\title{
Quark Confinement in the Two-Dimensional Lattice Higgs-Villain Model ${ }^{\star}$
}

\author{
Robert B. Israel ${ }^{\star \star}$ and Chiara R. Nappi ${ }^{\star \star \star}$ \\ Harvard University, Cambridge, Massachusetts 02138, USA
}

\begin{abstract}
We prove quark confinement in the two-dimensional lattice HiggsVillain model in the weak coupling region by using a Kirkwood-Salsburg equations for unbounded spins.
\end{abstract}

\section{Introduction}

In [1] Polyakov pointed out the role that instantons might play in the problem of confinement in gauge theories by showing that in the $U(1)$ three-dimensional pure gauge theory in the weak coupling region, due to the instantons' effect, external charges are confined.

His argument also predicts charge confinement in the Abelian twodimensional Higgs model because of the presence there of the Nielson-Olessen vortices. The Wilson loop expectation value [2] has indeed been computed in this model $[3,4]$ by taking into account only the instantons' contribution to the path integral in the dilute gas approximation. The answer for the quark-antiquark potential is the typical one-instanton contribution $\sim e^{-1 / g^{2}}$ where $g$ is the gauge coupling constant.

We want to test these ideas on lattice gauge theories, which combine the advantage of a rigorous formulation with many of the features of the continuum theories. We consider therefore the two-dimensional Higgs-Villain model [5-8], which differs from the usual $x-y$ version adopted for lattice scalar electrodynamics by the substitution

$$
e^{-\frac{1}{T} \cos \theta} \rightarrow \sum_{m \in \mathbb{Z}} e^{-\frac{1}{2 T}(\theta-2 \pi m)^{2}} .
$$

In the weak coupling region, the one we are going to investigate $(T \rightarrow 0)$, the above two functions are practically identical. Nevertheless the latter version is

* Research partially supported by the National Science Foundation under Grant PHY-77-18762

$\star \star$ On leave from Department of Mathematics, University of British Columbia, Vancouver, B.C., Canada. Research partially supported by National Research Council under Grant A4015

$\star \star \star$ On leave from Istituto di Fisica Teorica, Napoli, Italy 
more suitable for the use of duality transformation methods. In fact the strategy of our proof is to use the duality transformation to eliminate from the action the spin waves and isolate the vortices. We are able to give an upper and lower bound on the expectation of the Wilson loop in terms of the ratio of two partition functions in the vortices variables, one of which has an imaginary external field $[3,4]$. In the statistical mechanics language, we deal with a system of unbounded discrete spins in an exponentially decaying potential. For this system we work out a KirkwoodSalsburg equation and prove that it has solution in any finite (or infinite) volume for $g^{2}$ smaller and $\frac{g^{2}}{T}$ larger than given constants. Then we are able to compare Mayer series associated with the above mentioned partition functions and prove that, in this range of parameters, the dominant contribution to the difference comes from the one-vortex configurations. This will prove the confinement, with the predicted potential, of two external test charges, as long as they are fractionally charged to avoid binding with the Higgs field.

For previous rigorous results on quark confinement in lattice gauge theories see [9-13]. For the Higgs-Villain model, in particular, quark confinement is proved in [14] for $g^{2}$ sufficiently large, as communicated privately to us by one of the authors. High and low temperature expansions are also worked out.

\section{The Model}

We define

$$
d \sigma_{\Lambda}=\prod_{x \in A} \frac{d \varphi_{x}}{2 \pi} \prod_{\substack{r \mu \\ x \in A}} \frac{d A_{x \mu}}{2 \pi}
$$

and

$$
\begin{aligned}
e^{h_{\Lambda}}= & \prod_{\substack{x \mu \\
x \in \Lambda}} \sum_{m_{x \mu} \in \mathbb{Z}} \exp \left[-\frac{1}{2 T}\left(\varphi_{x+\hat{\mu}}-\varphi_{x}-q A_{x \mu}-2 \pi m_{x \mu}\right)^{2}\right] \\
& \cdot \prod_{x^{\prime} \in \Lambda} \sum_{m_{x^{\prime} \in \mathbb{Z}}} \exp \left[-\frac{1}{2 g^{2}}\left(A_{x^{\prime}}-2 \pi m_{x^{\prime}}\right)^{2}\right]
\end{aligned}
$$

where $\varphi_{x}$, the Higgs field, is an angle variable associated with the lattice site $x ; A_{x \mu}$, the gauge field, is an angle variable associated with the link between neighboring sites $x$ and $x+\hat{\mu}\left(\hat{\mu}, \hat{v}\right.$ being the unit displacements on the lattice) and $A_{x^{\prime}}$ (with prime we indicate the points in the dual lattice) is the "plaquette" variable defined, as usual, by

$$
A_{x^{\prime}}=A_{x \mu}+A_{x+\hat{\mu} \nu}-A_{x+\hat{v} \mu}-A_{x v} .
$$

We assume periodic boundary conditions. The partition function is

$$
Z_{\Lambda}=\int_{-\pi}^{\pi} d \sigma_{\Lambda} e^{h_{\Lambda}}
$$

and the Wilson loop expectation value that we are going to estimate is

$$
\left\langle\exp \left(i q^{\prime} \bigotimes_{\partial C} A_{x \mu}\right)\right\rangle=Z_{\Lambda}^{-1} \int_{-\pi}^{\pi} \exp \left(i q^{\prime} \bigotimes_{\partial C} A_{x \mu}\right) e^{h_{\Lambda}} d \sigma_{\Lambda},
$$


Fig. 1

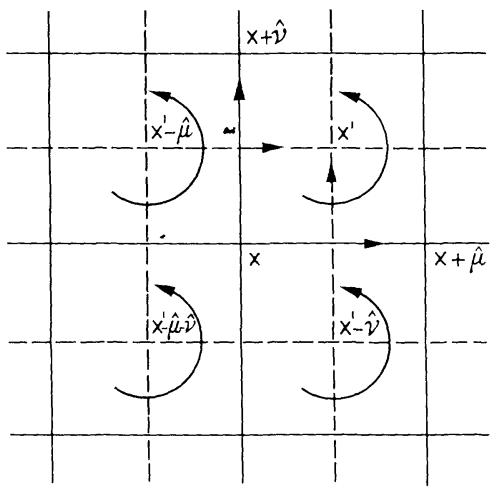

where $\partial C$ is an oriented closed path on the lattice with enclosed surface $C$. Above $q$ and $q^{\prime}$ are integer numbers which measure respectively the charge of the Higgs field and of the quark in units of the elementary charge $g$. We assume that the ratio $q^{\prime} / q$ is not an integer.

By using the Fourier transformation and the identity

$$
\sum_{n \in \mathbb{Z}} e^{i n 2 \pi x}=\sum_{n \in \mathbb{Z}} \delta(x-n)
$$

we get

$$
\begin{aligned}
& \sum_{m_{x \mu} \in \mathbb{Z}} \exp \left[-\frac{1}{2 T}\left(\varphi_{x+\hat{\mu}}-\varphi_{x}-q A_{x \mu}-2 \pi m_{x \mu}\right)^{2}\right] \\
& =\left(\frac{T}{2 \pi}\right)^{1 / 2} \sum_{m_{x \mu} \in \mathbb{Z}} \int_{-\infty}^{\infty} d \theta_{x \mu} \exp \left[-\frac{T}{2} \theta_{x \mu}^{2}+i \theta_{x \mu}\left(\varphi_{x+\mu}-\varphi_{x}-q A_{x \mu}-2 \pi m_{x \mu}\right)\right] \\
& =\left(\frac{T}{2 \pi}\right)^{1 / 2} \sum_{m_{x \mu} \in \mathbb{Z}} \exp \left[-\frac{T}{2} m_{x \mu}^{2}+i m_{x \mu}\left(\varphi_{x+\hat{\mu}}-\varphi_{x}-q A_{x \mu}\right)\right] .
\end{aligned}
$$

Analogously,

$$
\begin{aligned}
& \sum_{m_{x^{\prime} \in \mathbb{Z}}} \exp \left[-\frac{1}{2 g^{2}}\left(A_{x^{\prime}}-2 \pi m_{x^{\prime}}\right)^{2}\right] \\
& =\left(\frac{g^{2}}{2 \pi}\right)^{1 / 2} \sum_{m_{x^{\prime} \in \mathbb{Z}}} \exp \left[-\frac{g^{2}}{2} m_{x^{\prime}}^{2}+i m_{x^{\prime}} A_{x^{\prime}}\right] .
\end{aligned}
$$

We perform in (1.1) and (1.2) the integration over the $A_{x \mu}$ variables. In (1.1) we get the constraint (see Fig. 1)

$$
q \varepsilon_{\alpha \beta} m_{x \beta}=\partial_{\alpha} m_{x^{\prime}}
$$

where $\alpha=\mu, v, \beta=\mu, v, \varepsilon_{\mu v}$ is the completely antisymmetric tensor and $\partial_{\alpha} m_{x^{\prime}}=m_{x^{\prime}}-m_{x^{\prime}-\hat{\alpha}}$.

Now we suppose the Wilson loop oriented in such a way that

$$
\sum_{\partial \mathrm{C}} A_{x \mu}=-\sum_{x^{\prime} \in C} A_{x^{\prime}}=-\sum_{x^{\prime} \in A} \sigma_{x^{\prime}} A_{x^{\prime}},
$$


where $\sigma_{x}$ is the characteristic function of the region $C$. Then, in the numerator of (1.2), instead of (1.4) we get

$$
q \varepsilon_{\alpha \beta} m_{x \beta}=\partial_{\alpha} m_{x^{\prime}}-q^{\prime} \partial_{\alpha} \sigma_{x^{\prime}} .
$$

The integration over the $\varphi_{x}$ variables will give a constraint on the $m_{x \mu}$ 's of the form $\boldsymbol{V} \cdot \mathbf{m}=0$ which is automatically satisfied if (1.4) or (1.5) are satisfied. (The reason is that actually the $\varphi_{x}$ fields can be "gauged out" from the action, which is invariant under the local gauge transformation

$$
\varphi_{x} \rightarrow \varphi_{x}+q k_{x} \text { and } A_{x \mu} \rightarrow A_{x \mu}-k_{x}+k_{x+\hat{\mu}}
$$

for any function $k_{x}$.)

Because the $m_{x \mu}$ 's are integers we get from (1.4) the constraint

$$
m_{x^{\prime}}=q n_{x^{\prime}}+\theta
$$

and from (1.5)

$$
m_{x^{\prime}}=q n_{x^{\prime}}+q^{\prime} \sigma_{x^{\prime}}+\theta
$$

where $n_{x^{\prime}}$ takes integer values and $\theta$ is an integer $0 \leqq \theta \leqq q-1$. Therefore (1.2) becomes

$$
\left\langle\exp \left(i q^{\prime} \sum_{\partial C} A_{x \mu}\right)\right\rangle_{\Lambda}=\frac{\sum_{\theta=0}^{q-1} \tilde{Z}_{\Lambda}(\theta)}{\sum_{\theta=0}^{q-1} Z_{\Lambda}(\theta)},
$$

where

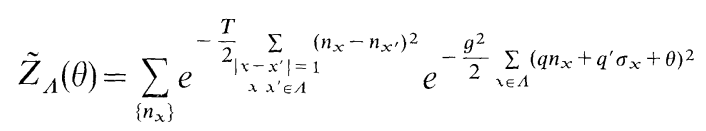

and

$$
Z_{\Lambda}(\theta)=\sum_{\left\{n_{x}\right\}} e^{-\frac{T}{2} \sum_{\substack{\left|x-x^{\prime}\right|=1 \\ \lambda, \nu^{\prime} \in \Lambda}}\left(n_{x}-n_{x^{\prime}}\right)^{2}} e^{-\frac{g^{2}}{2} \sum_{x \in \Lambda}\left(q n_{x}+\theta\right)^{2}}
$$

where now both $x$ and $x^{\prime}$ are sites in the dual lattice. By $\left\{n_{x}\right\}$ we mean the set of all configurations of integers in $\Lambda$. Because the above Gibbs measures are reflection positive, one gets by a straightforward application of chessboard estimates [15]

$$
\tilde{Z}_{\Lambda}(\theta) \leqq Z_{\Lambda}\left(q^{\prime}+\theta\right)^{\frac{|C|}{|\Lambda|}} Z_{\Lambda}(\theta)^{1-\frac{|C|}{|\Lambda|}}
$$

The main estimate of this paper (see Sect. 3) is

$$
\frac{Z_{\Lambda}(\theta)}{Z_{\Lambda}(0)} \leqq e^{-\alpha|\Lambda|}
$$

where $\alpha$ is a constant independent of $\Lambda$. We are assuming the ratio $\theta / q$ not to be an integer (otherwise obviously $Z_{\Lambda}(\theta) / Z_{\Lambda}(0) \equiv 1$ ). As a consequence of (1.10) and (1.9) the only contributions in (1.6), in the limit of large $A$, come from terms with $\theta=0$, 
namely

$$
\left\langle\exp \left(i q^{\prime} \sum_{\partial C} A_{x \mu}\right)\right\rangle_{\Lambda}=\frac{\sum_{\theta=0}^{q-1} \tilde{Z}_{\Lambda}(\theta)}{\sum_{\theta=0}^{q-1} Z_{\Lambda}(\theta)} \underset{\Lambda \rightarrow \infty}{\longrightarrow} \frac{\tilde{Z}_{\Lambda}(0)}{Z_{\Lambda}(0)} .
$$

We will always suppose to be at $\Lambda$ large enough to be authorized to use this limit expression. We point out that the above $\theta$ variables represent the discrete version of the $\theta$-vacua of the continuum Abelian Higgs model in 2 dimensions. The formula (1.11) just means that in the statistical mixture of all the $\theta$-vacua the $\theta=0$ vacuum has the largest weight. To pick up a particular $\theta \neq 0$ we must impose over our theory boundary conditions which correspond to fix non-zero background electrical field. Such would be a larger Wilson loop of charge $\theta q$. Then a slight modifications of the argument which led to (1.11) would give for the thermodynamic limit of the Wilson loop the value $\frac{\tilde{Z}_{\Lambda}(\theta)}{Z_{\Lambda}(\theta)}$. Alternatively we can simply restrict the ensemble to a particular value of $\theta$. For a more complete approach to the $\theta$ vacua in this model see $[7,14]$. In the appendix we will prove the following theorem:

Theorem 1. There is a constant $k>0$ (depending only on $T, g, q$ and $\left.q^{\prime}\right)$ such that

$$
k^{|\hat{\partial} C|}\left(\frac{Z_{\Lambda}\left(\theta+q^{\prime}\right)}{Z_{\Lambda}(\theta)}\right)^{\frac{|C|}{|\Lambda|}} \leqq\left(\frac{\tilde{Z}_{\Lambda}(\theta)}{Z_{\Lambda}(\theta)}\right) \leqq\left(\frac{Z_{\Lambda}\left(\theta+q^{\prime}\right)}{Z_{\Lambda}(\theta)}\right)^{\mid \frac{|C|}{|\Lambda|}} .
$$

Therefore, the estimate (1.10) will also prove the Wilson area law on (1.11) and will give an upper and lower bound on the confinement potential (for the sake of simplicity, we will restrict ourselves to the case $\theta=0$ ). We point out the importance of the requirement $q^{\prime} / q \notin \mathbb{Z}$. For $q^{\prime} / q \in \mathbb{Z}$ (1.12) shows that we detect only a length law. The last step is to use again the Poisson summation formula on $Z_{\Lambda}\left(q^{\prime}\right) / Z_{\Lambda}(0)$ and eliminate entirely from it the spin-wave variables with the usual technique of gaussian integration. The final answer is

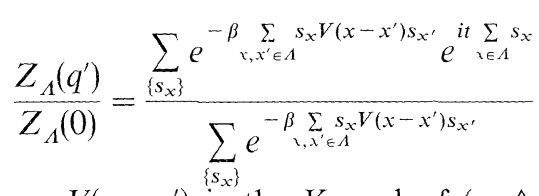

where $V\left(x-x^{\prime}\right)$ is the Kernel of $\left(-\Delta_{A}+m^{2}\right)^{-1}$ with $-\Delta_{A}$ the finite difference Laplacian chosen with periodic boundary conditions, $\beta=\frac{2 \pi^{2}}{T}, m^{2}=\frac{g^{2} q^{2}}{T}$, $t=-\frac{2 \pi q^{\prime}}{q}$. With $s_{x}$ we denote the vortices variables (taking values in $\mathbb{Z}$ ).

The strategy now is to compare the two free energies $p_{\Lambda}(t)=\frac{1}{|\Lambda|} \log Z_{\Lambda}\left(q^{\prime}\right)$ and $p_{\Lambda}=\frac{1}{|\Lambda|} \log Z_{\Lambda}(0)$ and prove that, uniformly in $\Lambda, p_{\Lambda}>p_{\Lambda}(t)$ for some opportune range of parameters $\beta$ and $m$. This will prove (1.10) and the Wilson area law. 


\section{The Kirkwood-Salsburg Equation for Unbounded Spins}

We will now formulate a Kirkwood-Salsburg [16] equation which applies to lattice models with unbounded spins. Because we desire estimates on (1.7) for a finite volume, we will not bother to take an infinite volume limit, although this could be done (see $[17,18]$ ). We assume that at each site $x$ of the finite lattice $\Lambda$ there is a spin $s_{x}$ taking integer values. The Hamiltonian will be

$$
H_{\Lambda}=\sum_{x, y \in \Lambda} s_{x} V(x-y) s_{y},
$$

the partition function $Z_{\Lambda}=\sum_{\left\{s_{x}\right\}} e^{-\beta H_{A}\left(\left\{S^{x}\right\}\right)}$ and the Gibbs equilibrium state

$$
\langle A\rangle_{A}=Z_{A}^{-1} \sum_{\left\{s_{x}\right\}} A\left(\left\{s_{x}\right\}\right) e^{-\beta H_{A}\left(\left\{s_{x}\right\}\right)} .
$$

The potential $V(x-y)$ is assumed to be real, symmetric, translationally invariant and positive definite with

$$
\sum_{x, y \in \Lambda} s_{x} V(x-y) s_{y} \geqq \varepsilon \sum_{x \in A} s_{x}^{2}
$$

for some constant $\varepsilon>0$, so the above sum over configurations $\left\{s_{x}\right\}$ converges absolutely. In order to express the equilibrium state as a perturbation of the "vacuum state" where all spins are 0 , we introduce a complex "activity" $z$, with

$$
Z_{\Lambda}(z)=\sum_{\left\{s_{x}\right\}} z^{\sum s_{x}^{2}} e^{-\beta H_{\Lambda}(\{s x\})}
$$

and [assuming $\left.Z_{\Lambda}(z) \neq 0\right]$

$$
\langle A\rangle_{\Lambda}(z)=Z_{\Lambda}(z)^{-1} \sum_{\left\{s_{x}\right\}} A\left(\left\{s_{x}\right\}\right) z^{\sum s_{x}^{2}} e^{-\beta H_{\Lambda}\left(\left\{s_{x}\right\}\right)} .
$$

The variable $z$ will sometimes be suppressed. It will also be convenient to introduce some notation. $Q_{X}$ will always denote a configuration $\left\{s_{x}\right\}_{x \in X}$ defined on $X \subset \Lambda$, with all $s_{x} \neq 0$. The set of all such configurations for all $X \subset \Lambda$ will be denoted by $Q$; note that this includes an empty configuration $\emptyset$ for the case $X=\emptyset$. We will sometimes join two or more configurations such as $Q_{X} Q_{Y}$, where the sets $X$ and $Y$ will always be disjoint. We write $v\left(Q_{X}\right)=\sum_{x \in X} s_{x}^{2}$ and $H\left(Q_{X}\right)=\sum_{x, y \in X} s_{x} V(x-y) s_{y}$. For any constant $r>0$, a norm on complex-valued functions on $Q$ is defined by $\|\varphi\|_{r}=\sup _{X, Q_{X}}\left|\varphi\left(Q_{X}\right)\right| e^{r v\left(Q_{X}\right)}$. The Banach space of functions $\varphi\left(Q_{X}\right)$ with $\|\varphi\|_{r}<\infty$ will be denoted $F_{r}$. In particular, the equilibrium state determines a function $\varrho_{A}$ on $Q$, where $\varrho_{\Lambda}\left(Q_{X}\right)$ is the probability that the configuration of spins in $X$ is $Q_{X}$. It can be expressed as

$$
\varrho_{\Lambda}\left(Q_{X}\right)=Z_{\Lambda}(z)^{-1} \sum_{Y \subset \Lambda \backslash X} \sum_{Q_{Y}} z^{v\left(Q_{X} Q_{Y}\right)} e^{-\beta H\left(Q_{X} Q_{Y}\right)} .
$$


Note that $\varrho_{\Lambda} \in F_{r}$ if $1 \leqq r \leqq \beta \varepsilon,|z| \leqq 1$ and $Z_{\Lambda}(z) \neq 0$, since

$$
\begin{aligned}
\left|\varrho_{\Lambda}\left(Q_{X}\right)\right| e^{r v\left(Q_{X}\right)} & \leqq\left|Z_{\Lambda}(z)\right|^{-1} \sum_{Y \subset \Lambda \backslash X} \sum_{Q_{Y}} e^{r v\left(Q_{X}\right)} e^{-\beta \varepsilon v\left(Q_{X} Q_{Y}\right)} \\
& \leqq\left|Z_{\Lambda}(z)\right|^{-1} \prod_{y \in \Lambda \backslash X} \sum_{t \in \mathbb{Z}} e^{-\beta \varepsilon t^{2}}
\end{aligned}
$$

and $\sum_{t \in \mathbb{Z}} e^{-\beta \varepsilon t^{2}}<2$ for $\beta \varepsilon \geqq 1$.

If $X \neq \emptyset$ and $x \in X$, let $X^{\prime}=X-\{x\}$, and let $Q_{X^{\prime}}$ be the restriction of $Q_{X}$ to $X^{\prime}$. Then

$$
\varrho_{\Lambda}(Q)=Z_{\Lambda}(z)^{-1} z^{s_{x}^{2}} e^{-\beta W^{x}\left(Q_{X}\right)} \sum_{Y \subset \Lambda \backslash X} \sum_{Q_{Y}} z^{v\left(Q_{X^{\prime}} Q_{Y}\right)} \cdot e^{-\beta H\left(Q_{X}, Q_{Y}\right)} e^{-2 \beta s_{x} \sum_{y \in Y} V(x-y) s_{y}}
$$

where $W^{x}\left(Q_{X}\right)=V(0) s_{x}^{2}+2 s_{x} \sum_{y \in X^{\prime}} V(x-y) s_{y}$. Now

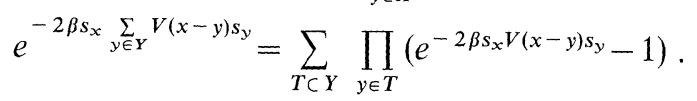

Letting $S=Y \backslash T$, we can rearrange terms to obtain

$$
\begin{aligned}
\varrho_{\Lambda}\left(Q_{X}\right)= & Z_{\Lambda}(z)^{-1} z^{s_{x}^{2}} e^{-\beta W^{x}\left(Q_{X}\right)} \sum_{T \subset \Lambda \backslash X} \sum_{Q_{T}} \sum_{S \subset A \backslash(X \cup T)} \sum_{Q_{S}} z^{v\left(Q_{X} \cdot Q_{T} Q_{S}\right)} \\
& \cdot k\left(s_{x}, Q_{T}\right) e^{-\beta H\left(Q_{X}, Q_{T} Q_{S}\right)}
\end{aligned}
$$

where $k\left(s_{x}, Q_{T}\right)=\prod_{y \in T}\left(e^{-2 \beta s_{x} V(x-y) s_{y}}-1\right)$; note $k\left(s_{x}, \emptyset\right)=1$. Performing the sum over $S$ and $Q_{S}$, we obtain

$$
\varrho_{\Lambda}\left(Q_{X}\right)=z^{s_{x}^{2}} e^{-\beta W^{x}\left(Q_{X}\right)} \sum_{T \subset \Lambda \backslash X} \sum_{Q_{T}} k\left(s_{x}, Q_{T}\right) R_{x} \varrho_{\Lambda}\left(Q_{X^{\prime}} Q_{T}\right)
$$

where $R_{x} \varphi\left(Q_{Y}\right) \equiv \varphi\left(Q_{Y}\right)-\sum_{t \neq 0} \varphi\left(t_{x} Q_{Y}\right)$ represents the probability of having spins 0 at $x$ and $Q_{Y}$ on $Y$. The Eq. (2.3) together with

$$
\varrho_{\Lambda}(\emptyset)=1
$$

constitute our Kirkwood-Salsburg equations for suitable choices of $x$. Formulas (2.3)-(2.4) can be expressed as $\varrho_{A}=\delta+K_{\Lambda} \varrho_{\Lambda}$ where $\delta(\emptyset)=1, \delta\left(Q_{X}\right)=0$ for nonempty $Q_{X}$, and

$$
\begin{aligned}
& K_{\Lambda} \varphi(\emptyset)=0, \\
& K_{\Lambda} \varphi\left(Q_{X}\right)=z^{s_{x}^{2}} e^{-\beta W^{x}\left(Q_{X}\right)} \sum_{T \subset \Lambda \backslash X} \sum_{Q_{T}} k\left(s_{x}, Q_{T}\right) R_{x} \varphi\left(Q_{X^{\prime}} Q_{T}\right) .
\end{aligned}
$$

We want to show that under suitable conditions, $K_{A}$ takes $F_{r}$ to $F_{r}$ with $\left\|K_{\Lambda}\right\|<1$ for $|z| \leqq 1$. Then the equation $\varphi=\delta+K_{\Lambda} \varphi$ has a unique solution in $F_{r}$, which is analytic as a function of $z$ in a neighborhood of $|z| \leqq 1$, and can be expressed as a power series $\varphi=\left(1-K_{A}\right)^{-1} \delta=\sum_{n=0}^{\infty} K^{n} \delta$. If $1 \leqq r \leqq \beta \varepsilon$ this solution must be $\varrho_{A}\left[\right.$ if $\left.Z_{A}(z) \neq 0\right]$, since we know $\varrho_{A} \in F_{r}$. In fact, $Z_{\Lambda}(z) \neq 0$ for $|z| \leqq 1$; for any configuration $Q_{\Lambda}$ of nonzero spins in $A, e^{\beta H\left(Q_{A}\right)} z^{-v\left(Q_{\Lambda}\right)} \varphi\left(Q_{\Lambda}\right)$ is an analytic function of $z$ in $|z| \leqq 1$ and coincides with $Z_{\Lambda}(z)^{-1}$ where $Z_{\Lambda}(z) \neq 0$. 
Our basic result is the following

Theorem 2. Let $S_{1}=\sum_{x \neq 0}|V(x)|$ and $S_{2}=\sum_{x \neq 0} V(x)^{2}$. If $r \geqq 1$ and $L=\beta(2 \varepsilon-V(0))-r$ $-\beta^{2} \frac{S_{2}}{r}-2 \varrho \frac{S_{1}}{r} \geqq 0$, then for $|z| \leqq 1, K_{A}$ is an operator in $F_{r}$ with norm at most $2|z| e^{-L}$.

Proof: Let us choose the distinguished site $x=x\left(Q_{X}\right)$ in the definition of Eq. (2.5) to satisfy the inequality $W^{x}\left(Q_{X}\right) \geqq(2 \varepsilon-V(0)) s_{x}^{2}$. Note that there is always at least one $x \in X$ satisfying the above inequality, since

$$
\sum_{x \in X} W^{x}\left(Q_{X}\right)=2 H\left(Q_{X}\right)-V(0) \sum_{x \in X} s_{x}^{2} \geqq(2 \varepsilon-V(0)) \sum_{x \in X} s_{x}^{2} .
$$

We will suppose such a choice is made. Moreover,

$$
\begin{aligned}
&\left|R_{x} \varphi\left(Q_{X^{\prime}} Q_{T}\right)\right| \leqq\left(1+\sum_{t \neq 0} e^{-r t^{2}}\right) e^{-r v\left(Q_{X^{\prime}} Q_{T}\right)}\|\varphi\|_{r} \\
&<2 e^{-r v\left(Q_{X^{\prime}} Q_{T}\right)}\|\varphi\|_{r} \quad \text { if } \quad r \geqq 1 .
\end{aligned}
$$

Thus [using (2.6)]

$$
\begin{aligned}
& \left|K_{\Lambda} \varphi\left(Q_{X}\right)\right| e^{r v\left(Q_{x}\right)} \\
& \leqq 2|z|^{s_{x}^{2}} e^{-\beta(2 \varepsilon-V(0)) s_{x}^{2}} e^{r s_{x}^{2}}\|\varphi\|_{r} \sum_{T \subset \Lambda \backslash X} \sum_{Q_{T}}\left|k\left(s_{x}, Q_{T}\right)\right| e^{-r v\left(Q_{T}\right)} .
\end{aligned}
$$

We claim that

$$
\sum_{T \subset \Lambda} \sum_{Q_{T}}\left|k\left(s_{x}, Q_{T}\right)\right| e^{-r v\left(Q_{T}\right)} \leqq \exp \left(\frac{\beta^{2} S_{2}+2 \beta S_{1}}{r} s_{x}^{2}\right)
$$

which will prove the theorem.

In fact,

$$
\begin{aligned}
& \sum_{T \subset A \backslash\{x\}} \sum_{Q_{T}}\left|k\left(s_{x}, Q_{T}\right)\right| e^{-r v\left(Q_{T}\right)} \\
& =\prod_{y \neq x}\left(1+\sum_{t \neq 0} e^{-r t^{2}}\left|e^{-2 \beta s_{x} V(x-y) t}-1\right|\right) \\
& =\prod_{y \neq x}\left(1+\sum_{t=1}^{\infty} e^{-r t^{2}}\left(e^{2 \beta\left|s_{x} V(x-y)\right| t}-e^{-2 \beta\left|s_{x} V(x-y)\right| t}\right)\right. \\
& =\prod_{y \neq x}\left(1+e^{\alpha(y)^{2} r} f(\alpha(y))\right) .
\end{aligned}
$$

where $\alpha(y)=\frac{\beta\left|s_{x} V(x-y)\right|}{r}$ and $f(\alpha)=\sum_{t=1}^{\infty}\left(e^{-r(t-\alpha)^{2}}-e^{-r(t+\alpha)^{2}}\right)$. Note that for $r \geqq 1$ we have $f(\alpha) \leqq 2 \alpha$ for all $\alpha \geqq 0$. Now $1+e^{\alpha^{2} r} f(\alpha) \leqq e^{\alpha^{2} r}(1+2 \alpha) \leqq e^{\alpha^{2} r+2 \alpha}$ so that

$$
\begin{aligned}
\sum_{T \subset A \backslash\{x\}} \sum_{Q_{T}}\left|k\left(s_{x}, Q_{T}\right)\right| e^{-r v\left(Q_{T}\right)} & \leqq \exp \sum_{y \neq x}\left(\alpha(y)^{2} r+2 \alpha(y)\right) \\
& =\exp \left(\beta^{2} \frac{S_{2}}{r} s_{x}^{2}+2 \beta \frac{S_{1}}{r}\left|s_{x}\right|\right)
\end{aligned}
$$

which completes the proof since $\left|s_{x}\right| \leqq s_{x}^{2}$. 
Corollary. Suppose $2 \varepsilon-V(0)>2 \sqrt{S_{2}}$. Then for any $L_{0}>0$ there is a $\beta_{0}$ [depending on $V(0), S_{1}, S_{2}$, and $\left.L_{0}\right]$ such that if $\beta>\beta_{0}$ then $K_{A}$ has norm at most $2|z| e^{-L} 0$ in some $F_{r}$ with $1 \leqq r \leqq \beta \varepsilon$. In fact we can take $r=\beta(\varepsilon-V(0) / 2)$.

Proof. If $\quad r=\beta r_{0} \quad$ then $\quad L=\beta\left(2 \varepsilon-V(0)-r_{0}-\frac{S_{2}}{r_{0}}\right)-\frac{2 S_{1}}{r_{0}}$. So $L \geqq L_{0} \quad$ if $2 \varepsilon-V(0)-r_{0}-\frac{S_{2}}{r_{0}}>0$ and $\beta>\frac{L_{0}+2 S_{1} * r_{0}}{2 \varepsilon-V(0)-r_{0}-S_{2} * r_{0}} \equiv \beta_{0}$. The condition on $r_{0}$ can be satisfied if and only if $2 \varepsilon-V(0)>2 \sqrt{S_{2}}$ and if so, $r_{0}=\varepsilon-V(0) / 2$ will do.

\section{Application to the Model}

In application to the model of Sect. 1 , we take $V(x)=\left\langle e_{x},\left(\triangle_{A}+m^{2}\right)^{-1} e_{0}\right\rangle$ where $-\triangle_{A}$ is the finite-difference Laplacian with periodic boundary conditions (i.e. $-\triangle_{\Lambda} f(x)=\sum_{\substack{|x-y|=1 \\ y \in \Lambda}}(f(x)-f(y))$ where $\Lambda=\mathbb{Z}^{2} / M \mathbb{Z}^{2}$ for some integer $\left.M\right) \cdot e_{x}$ is the function $e_{x}(y)=\delta_{x y}$ and $\langle$,$\rangle is the inner product in l^{2}(\Lambda)$. Note the following properties of $V(x-y)$ :

(a) Since $0 \leqq-\triangle_{\Lambda} \leqq 8$ we have $\frac{1}{m^{2}+8} \leqq\left(-\triangle_{A}+m^{2}\right)^{-1} \leqq \frac{1}{m^{2}}$. Thus $\varepsilon=\frac{1}{m^{2}+8}$ and $V(0) \geqq \frac{1}{m^{2}+8}$. Actually $V(0)=\left\langle e_{0},\left(-\Delta_{A}+m^{2}\right)^{-1} e_{0}\right\rangle \geqq\left\langle e_{0},\left(-\Delta_{A}+m^{2}\right) e_{0}\right\rangle^{-1}$ $=\frac{1}{m^{2}+4}$.

(b) $V(x) \geqq 0$ for all $x$. For if not, there would be some $y$ with $V(y)=\inf _{x} V(x)<0$. Since $y \neq 0$ and $\left(-\triangle_{\Lambda}+m^{2}\right) V(x)=e_{x}$,

$$
V(y)=\frac{1}{4+m^{2}} \sum_{\left|y^{\prime}-y\right|=1} V\left(y^{\prime}\right) \geqq \frac{4}{4+m^{2}} V(y)
$$

which contradicts $V(y)<0$.

(c) $\sum_{y \in \Lambda} V(y)=\left\langle\mathbb{1},\left(-\triangle_{\Lambda}+m^{2}\right)^{-1} e_{0}\right\rangle=\frac{1}{m^{2}}$ since $\triangle_{\Lambda} \mathbb{1}=0$ (where $\mathbb{1}$ is the constant function 1 on $\Lambda$ ). Thus $S_{1}=\frac{1}{m^{2}}-V(0) \leqq \frac{4}{m^{4}}$ and $S_{2} \leqq S_{1}^{2} \leqq \frac{16}{m^{8}}$. So the condition $2 \varepsilon-V(0)>2 \sqrt{S_{2}}$ is satisfied for $m$ sufficiently large. Using more careful estimates, we can in fact prove that it holds for $m^{2} \geqq 6.7$. Finally, we note that $\frac{\beta_{0}}{m^{2}} \rightarrow L_{0}+1$ as $m \rightarrow \infty$ (taking $r=1$ ). Therefore in this limit the condition $\beta>\beta_{0}$ reads $\frac{2 \pi^{2}}{q^{2} g^{2}}>L_{0}+1$ (see Fig. 2).

Now, in order to deal with (1.10) we define $Z_{A}(z, t),\langle\rangle_{A}(z, t), \varrho_{A}(z, t)$ and $K_{A}(z, t)$ by replacing $z^{s_{x}^{2}}$ by $z^{s_{x}^{2}} e^{i t s_{x}}$ throughout. This will not affect any of our estimates. 


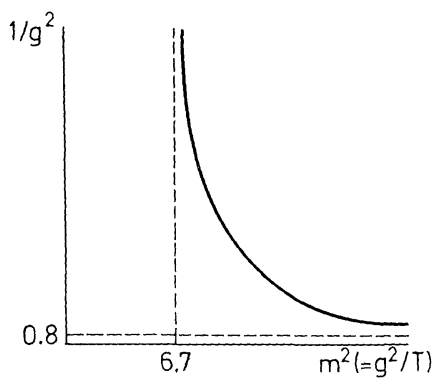

Fig. 2. Region of convergence of the K.S. equation for $q=3$

Let us define the pressures $p_{\Lambda}(z)=\frac{1}{|\Lambda|} \log Z_{A}(z)$ and $p_{A}(z, t)=\frac{1}{|\Lambda|} \ln Z_{\Lambda}(z, t)$. Our goal is to show that $p_{A}(1)-p_{A}(1, t)\left[\equiv p_{A}-p_{A}(t)\right.$ of Sect. 1] is bounded below by some positive number independent of $\Lambda$. Note that $p_{\Lambda}(0)=p_{A}(0, t)$ and $\frac{d}{d z} p_{\Lambda}(z)=\frac{1}{z|\Lambda|} \sum_{x \neq \Lambda}\left\langle s_{x}^{2}\right\rangle_{\Lambda}(z) \quad$ where $\left\langle s_{x}^{2}\right\rangle_{\Lambda}=\sum_{s_{x} \neq 0} s_{x}^{2} \varrho_{\Lambda}\left(s_{x}\right) ;$ similarly $\frac{d}{d z} p_{\Lambda}(z, t)$ $=\frac{1}{z|\Lambda|} \sum_{x \in \Lambda}\left\langle s_{x}^{2}\right\rangle_{\Lambda}(z, t)$. Therefore

$$
p_{\Lambda}(1)-p_{\Lambda}(1, t)=\int_{0}^{1} \frac{d z}{z} \frac{1}{|\Lambda|} \sum_{x \in A}\left(\left\langle s_{x}^{2}\right\rangle_{\Lambda}(z)-\left\langle s_{x}^{2}\right\rangle_{\Lambda}(z, t)\right) .
$$

Using the power series $\varrho_{A}(z)=\sum_{n=0}^{\infty} K_{\Lambda}^{n}(z) \delta$ and $\varrho_{A}(z, t)=\sum_{n=0}^{\infty} K_{A}^{n}(z, t) \delta$ and evaluating the $n=1$ terms explicitly, we have

$$
\begin{aligned}
\left\langle s_{x}^{2}\right\rangle_{\Lambda}(z)-\left\langle s_{x}^{2}\right\rangle_{A}(z, t)= & 2 \sum_{s_{x}=1}^{\infty} s_{x}^{2} z^{s_{x}^{2}} e^{-\beta V(0) s_{x}^{2}}\left(1-\cos \left(s_{x} t\right)\right) \\
& +\sum_{n=2}^{\infty} \sum_{s_{x} \neq 0} s_{x}^{2}\left(K_{\Lambda}^{n}(z) \delta\left(s_{x}\right)-K_{\Lambda}^{n}(z, t) \delta\left(s_{x}\right)\right) .
\end{aligned}
$$

Note that $K_{\Lambda} \delta\left(Q_{X}\right)=0$ if $|X| \neq 1$, so $\left\|K_{\Lambda}(z) \delta\right\|=\left\|K_{A}(z, t) \delta\right\|=|z| e^{r-\beta V(0)}$. Thus

$$
\left|K_{A}^{n+1} \delta\left(s_{x}\right)\right| \leqq e^{-r s_{x}^{2}}\left\|K_{A}\right\|^{n}\left\|K_{A} \delta\right\| \leqq|z| e^{r\left(1-s_{x}^{2}\right)} e^{-\beta V(0)}\left(2|z| e^{-L}\right)^{n},
$$

and

$$
\begin{aligned}
& \sum_{n=2}^{\infty} \sum_{s_{x} \neq 0} s_{x}^{2}\left(K_{\Lambda}^{n}(z) \delta\left(s_{x}\right)-K_{\Lambda}^{n}(z, t) \delta\left(s_{x}\right)\right) \\
& \leqq \frac{4|z|^{2} e^{-\beta V(0)-L}\left(\sum_{s \neq 0} s^{2} e^{r\left(1-s^{2}\right)}\right)}{1-2|z| e^{-L}} \\
& \leqq 18|z|^{2} e^{-\beta V(0)-L} \text { if } \quad r \geqq 1 \text { and } 2|z| e^{-L}<\frac{1}{2} .
\end{aligned}
$$


Thus from (3.1) and (3.2)

$$
p_{\Lambda}(1)-p_{\Lambda}(1, t) \geqq 2 e^{-\beta V(0)}(1-\cos t)-18 e^{-\beta V(0)-L},
$$

which is $>0$ if $L>L_{0}=\log \frac{9}{1-\cos 2 \pi q^{\prime} / q}$.

By the corollary of the previous section and the remark (c) this condition is satisfied in a region of the $\left(\beta, m^{2}\right)$ plane which is asymptotic to the lines $m^{2}=6.7$ and $\beta / m^{2}=L_{0}+1$.

Finally, we note that from (3.2) as $\beta \rightarrow \infty$, the coefficient in our "area law" is asymptotically $2 e^{-\beta V(0)}\left(1-\cos 2 \pi q^{\prime} / q\right)$ where $\beta V(0)$ is approximately $\beta / m^{2}=2 \pi^{2} / g^{2} q^{2}$ for large $m$. As already mentioned, this is the same expression computed in the dilute gas approximation for the confinement potential in the continuum Abelian Higgs model in the $\theta=0$ vacuum. Analogously working in a $\theta \neq 0$ vacuum, we would have obtained the more general formula $2 e^{-\beta V(0)}\left(\cos \theta-\cos \left(\theta+2 \pi q^{\prime} / q\right)\right)$.

Acknowledgments. We acknowledge very helpful conversations with M. Peskin, E. Seiler, T. Spencer, P. Steinhard and E. Witten. We thank A. Jaffe for his kind hospitality at the Lyman Laboratory of Physics.

\section{Appendix}

Proof of Theorem 1. The upper bound of $\frac{\tilde{Z}_{\Lambda}(\theta)}{Z_{\Lambda}(\theta)}$ is (1.9). The method for obtaining the lower bound is basically contained in Lebowitz and Presutti [17]. Since they assume translation invariance (which we do not have due to the Wilson loop), and since some simplifications are possible with our model, we will present the details below. First we want to show that $\left\langle n_{x}^{2}\right\rangle_{A}$ is bounded uniformly in $x, A$ and $C$ where $\langle\cdot\rangle_{A}$ is the expectation corresponding to $\tilde{Z}_{\Lambda}(\theta)$. More generally, for $h=\left\{h_{x}\right\}_{x \in A}$, we let $\langle\cdot\rangle_{h, A}$ be the expectation corresponding to

$$
Z_{\Lambda}(h, \theta)=\sum_{\left\{n_{x}\right\}} e^{-\frac{T}{2} \sum_{\left|x-x^{\prime}\right|=1}\left(n_{x}-n_{x^{\prime}}\right)^{2}} e^{-\frac{g 2}{2} \sum_{x \in A}\left(q n_{x}+h_{x}+\theta\right)^{2}}
$$

Let $P_{N}(n)=1$ if $|n| \geqq N, 0$ otherwise. If all $h_{x}+\theta \leqq 0$ (which we can always assure by subtracting a multiple of $q$ from $\theta$ ) the second GKS inequality (in the form of [19], Theorem VIII. 14 A) implies

$$
\frac{\partial\left\langle P_{N}\left(n_{x}\right)\right\rangle_{h, \Lambda}}{\partial h_{y}}=-g^{2}\left(\left\langle n_{y} P_{N}\left(n_{x}\right)\right\rangle_{h, \Lambda}-\left\langle n_{y}\right\rangle_{h, \Lambda}\left\langle P_{N}\left(n_{x}\right)\right\rangle_{h, \Lambda}\right) \leqq 0 .
$$

Thus,

$$
\left\langle P_{N}\left(n_{x}\right)\right\rangle_{h, A} \leqq\left\langle P_{N}\left(n_{x}\right)\right\rangle_{h^{\prime}, A} \quad \text { where all } \quad h_{x}^{\prime}=\min _{y \in A} h_{y} .
$$

Now by the chessboard estimate:

$$
\begin{aligned}
\left\langle P_{N}\left(n_{x}\right)\right\rangle_{h^{\prime}, \Lambda} & \leqq\left\langle\prod_{y \in \Lambda} P_{N}\left(n_{y}\right)\right\rangle_{h^{\prime}, \Lambda}^{1 /|\Lambda|} \\
& \left.\leqq \sum_{|n| \geqq N} e^{-\frac{g^{2}}{2}\left[\left(q n+h^{\prime}+\theta\right)^{2}-\right.} \inf _{m \in \mathbb{Z}}\left(q m+h^{\prime}+\theta\right)^{2}\right] \\
& \leqq e^{-\gamma N^{2}+\delta}
\end{aligned}
$$


for some $\gamma>0$ and $\delta$ depending only on $g^{2}, q, h^{\prime}$ and $\theta$. From this it is easy to see that

$$
\left\langle n_{x}^{2}\right\rangle_{h^{\prime}, \Lambda} \leqq 2 \gamma \int_{0}^{\infty} t^{3} e^{-\gamma t^{2}+\delta} d t \equiv M
$$

Now for any $X \subset A$

$$
\operatorname{Prob}\left(\sum_{x \in X} n_{x}^{2} \geqq N|X|\right) \leqq\left\langle\sum_{x \in X} n_{x}^{2}\right\rangle / N|X| \leqq M / N
$$

Choose $N>4 M$. Let $X_{1}$ (resp. $X_{2}$ ) be the set of sites in $C($ resp. $\Lambda \backslash C$ ) that have a nearest neighbor in $\Lambda \backslash C$ (resp. $C$ ). Let $B$ be the set of configurations $\left\{n_{x}\right\}_{x \in \Lambda}$ with $\sum_{x \in X_{i}} n_{x}^{2} \geqq N\left|X_{i}\right|$ for $i=1$ or 2 . Then we can write

$$
\tilde{\mathrm{Z}}_{\Lambda}(\theta)=\sum_{B} e^{-H}+\sum_{B^{c}} e^{-H(C)-H(A \backslash C)-\frac{T}{2} \sum_{\substack{x \in C \\ y \in A C \\|x-y|=1}}\left(n_{x}-n_{y}\right)^{2} .}
$$

Using $\left(n_{x}-n_{y}\right)^{2} \leqq 2\left(n_{x}^{2}+n_{y}^{2}\right)$ we have

$$
\begin{aligned}
\tilde{Z}_{\Lambda}(\theta) & \geqq e^{-4 N T\left|X_{1} \cup X_{2}\right|} \sum_{B^{c}} e^{-H(C)-H(\Lambda \backslash C)} \\
& \geqq \frac{9}{16} e^{-8 N T|\hat{\partial} C|} Z_{\Lambda \backslash C}(\theta) Z_{C}\left(\theta+q^{\prime}\right) .
\end{aligned}
$$

Similarly $Z_{\Lambda}(\theta) \leqq \frac{1}{2} Z_{\Lambda}(\theta)+e^{8 N T|\partial C|} Z_{\Lambda \backslash C}(\theta) Z_{C}(\theta)$

so

$$
\frac{\tilde{Z}_{\Lambda}(\theta)}{Z_{\Lambda}(\theta)} \geqq \frac{9}{32} e^{-16 N T|\hat{\partial} C|} \frac{Z_{C}\left(\theta+q^{\prime}\right)}{Z_{C}(\theta)} .
$$

Analogously

$$
\frac{Z_{\Lambda}\left(\theta+q^{\prime}\left(1-\sigma_{x}\right)\right)}{Z_{\Lambda}\left(\theta+q^{\prime}\right)} \geqq \frac{9}{32} e^{-16 N T|\partial C|} \frac{Z_{C}(\theta)}{Z_{C}\left(\theta+q^{\prime}\right)}
$$

while

$$
\frac{Z_{\Lambda}\left(\theta+q^{\prime}\left(1-\sigma_{x}\right)\right)}{Z_{\Lambda}\left(\theta+q^{\prime}\right)} \leqq\left[\frac{Z_{\Lambda}(\theta)}{Z_{\Lambda}\left(\theta+q^{\prime}\right)}\right]^{\frac{|C|}{|\Lambda|}}
$$

by the chessboard estimate. Putting these together,

$$
\frac{\tilde{Z}_{\Lambda}(\theta)}{Z_{\Lambda}(\theta)} \geqq \frac{81}{1024} e^{-32 N T|\hat{c} C|}\left[\frac{Z_{\Lambda}\left(\theta+q^{\prime}\right)}{Z_{A}(\theta)}\right]^{|C|}
$$

which completes the proof.

\section{References}

1. Polyakov, A. M.: Phys. Lett. 59 B, 82-84 (1975)

2. Wilson, K.: Phys. Rev. D 10, 2445-2459 (1974)

3. Callan, C., Dashen, R., Gross, D.J.: Phys. Lett. 66 B, 375-381 (1977) 
4. Coleman, S.: Erice Lectures (1977)

5. Villain, J. : J. Phys. (Paris) 36, 581-590 (1975)

6. José, J., Kadanoff, L., Kirkpatrick, S., Nelson, D. : Phys. Rev. B 16, 1217-1241 (1977)

7. Fröhlich, J.: Lecture notes of Rome Conference (1977)

8. Peskin, M.E.: Mandelstam-t'Hooft duality. Ann. Phys. (to appear)

9. Osterwalder, K., Seiler, E.: Ann. Phys. 110, 440-471 (1978)

10. Glimm, J., Jaffe, A.: Phys. Lett. 66 B, 67-69 (1977)

11. De Angelis, G.F., De Falco, D.: Lett. Nuovo Cimento 18, 536-538 (1977)

12. Challifour, J.L., Weingarten, D. : J. Math. Phys. 19, 1134-1136 (1978)

13. Gawedzki, K.: On confinement of fermions in strongly coupled lattice gauge theories. Preprint, Max-Planck-Institut (1978)

14. Brydges, D., Fröhlich, J., Seiler, E.: On the construction of quantized Gauge fields (to appear)

15. Fröhlich, J., Israel, R., Lieb, E.H., Simon, B. : Commun. math. Phys. 62, 1-34 (1978)

16. Ruelle, D.: Statistical mechanics. New York: W. A. Benjamin 1969

17. Lebowitz, J.L., Presutti, E.: Commun. math. Phys. 50, 195-218 (1976)

18. Kunz, H.: Commun. math. Phys. 59, 53-69 (1978)

19. Simon, B.: The $P(\Phi)_{2}$ Euclidean (quantum) field theory. Princeton, NJ : Princeton University Press 1974

Communicated by A. Jaffe

Received May 3, 1978

\section{Erratum}

Cantoni, V.: Enveloping Subspaces and Superposition of States. Commun. math. Phys. 50, 241-244 (1976)

p. 242, line 10 : Replace "Borel sets" by "closed sets". 